Gerichte vs. Gesetzgeber 


\title{
Politischer Druck durch Rechtsschutz - Auf dem Weg zur öffentlich-rechtlichen „Public Interest Litigation“?
}

\author{
Dr. Lutz Friedrich"
}

Abstract: Immer häufiger sehen sich die Verwaltungsgerichte mit Rechtsbehelfen konfrontiert, die in der Sache nicht die Verletzung subjektiver öffentlicher Rechte, sondern - unter Berufung auf (vermeintliche) Allgemeininteressen - Verstöße gegen objektives Recht monieren und weit über den konkreten Streitfall hinaus eine "politische Agenda"verfolgen. Dazu gehören viele der aufsehenerregenden „Klimaklagen“. Der Beitrag unternimmt den Versuch, Chancen und Risiken solcher Formen der sog. Public Interest bzw. Climate Change Litigation zu skizzieren und deren verfassungsrechtliche Grenzen auszuloten. Dabei geht es weniger um Detailfragen des Öffentlichen Prozessrechts als vielmehr um grundlegende Fragen des gerichtlichen Rechtsschutzes, der Gewaltenteilung und einer Privatisierung des gemeinen Wohls.

\section{Einführung: Durchsetzung politischer Ziele über den Rechtsweg}

Die Durchsetzung politischer Ziele und von Gemeinwohlbelangen über den Rechtsweg ist an sich kein neues Phänomen. Im Gegenteil: Dem individuellen, subjektiven Rechtsschutz eignet grundsätzlich eine objektive Dimension. Die einzelne Klägerin verteidigt zwar nach der „Strukturentscheidung“ des Art. 19 Abs. 4 S. 1 GG im Regelfall primär eigene Rechte ${ }^{1}$, verhilft aber zugleich der Rechtsordnung insgesamt - und damit der Rechtmäßigkeit staatlichen Handelns als einem zentralen Gemeinwohlbelang (Art. 1 Abs. 3, 20 Abs. 3, 28 Abs. 1 S. 1 GG) - zur praktischen Gel-

* Die Arbeiten an dem Manuskript wurden vor der Entscheidung des Bundesverfassungsgerichts über die Verfassungsbeschwerden gegen das Klimaschutzgesetz abgeschlossen (s.u. Fn. 10). Eine erweiterte Fassung des Vortrags ist von der DÖV zur Veröffentlichung angenommen worden.

$1 \mathrm{Zu}$ dieser „Systementscheidung“ im ersten Zugriff nur Schmidt-Aßmann, in: Herzog u.a. (Hrsg.), Maunz/Dürig GG, Art. 19 IV (Juli 2014) Rn. 8; Huber, in: ders./ Voßkuhle (Hrsg.), v. Mangoldt/Klein/Starck GG I, 7. Aufl. 2018, Art. 19 Rn.341, 347. BVerfG, NVwZ 2009, 240 (242); BVerfG, NVwZ 2009, 1426 (1427); BVerwGE 147, 312 (316, Rn. 18); E 154, 328 (332 f., Rn. 16). 
tung ${ }^{2}$. Zuletzt aber häufen sich fach- und speziell verwaltungsgerichtliche Verfahren, in denen die Rechtsbehelfsführerin ihr Rechtsschutzziel noch viel stärker auf die Durchsetzung vermeintlicher Allgemeininteressen konzentriert. Ihre eigenen Rechte - so ihr solche überhaupt zustehen - macht sie dabei allenfalls äußerst vordergründig geltend, faktisch werden sie durch das übergeordnete strategische Ziel sogar fast vollständig überlagert.

Das gilt handgreiflich für die Popular- und Verbandspopularklagen im Umwelt- und Naturschutzrecht ( $\$ 2$ Abs. 1 UmwRG und $\$ 64$ Abs. 1 BNatSchG) ${ }^{3}$. Sie sollen, obwohl sie der Dritten Gewalt folgenschwere Funktionserweiterungen aufdrängen ${ }^{4}$, hier weitgehend außer Betracht bleiben: Zum einen sind sie durch formelles Gesetz von der Geltendmachung subjektiver Rechte befreit. Zum anderen handelt es sich um klar abgrenzbare Ausnahmetatbestände. Diese sind noch dazu überwiegend durch das Unionsrecht indiziert, das ohnehin viel stärker als das deutsche Recht nach Legalität strebt und um seiner „effektiven Wirksamkeit“ willen die Einzelne ganz unabhängig von ihrer subjektiven Berechtigung dafür „mobilisiert“ (Art. 4 Abs. 3 EUV, Art. 19 Abs. 1 UAbs. 2 EUV, Art. 47 Abs. 1 ChGrEU) ${ }^{5}$. Das gilt zumindest für den Zugang zu den mitgliedstaatlichen Gerichten, während der EuGH die eigene „Tür" - zuletzt auch in Klimafragen - deutlich strikter reglementiert ${ }^{6}$.

Sub specie Demokratieprinzip und Gewaltengliederung viel mehr Konfliktpotential bergen dagegen diejenigen Konstellationen, in denen ohne eine solche gesetzliche Grundlage die Einzelne weniger ihre Rechte als vielmehr das Recht durchzusetzen sucht und sich dazu auf die treuhänderi-

2 Die auch-objektive Funktion des individuellen gerichtlichen Rechtsschutzes betonen etwa Schulze-Fielitz, in: Dreier (Hrsg.), GG I, 3. Aufl. 2013, Art. 19 IV Rn. 60; Huber (Fn. 1), Art. 19 Rn.343; Ernst, in: Kämmerer/Kotzur (Hrsg.), v. Münch/ Kunig GG I, 7. Aufl. 2021, Art. 19 Rn. 99.

3 Im ersten Zugriff Schoch, in: Hoffmann-Riem/Schmidt-Aßmann/Voßkuhle (Hrsg.), GVwR III, 2. Aufl. 2013, \50 Rn. 176 ff.; Kment, NVwZ 2018, 921 ff.; Schlacke, Umweltrecht, 7. Aufl. 2019, \$10 Rn. 65 ff.; Külpmann, DVBl. 2019, $140 \mathrm{ff}$.

4 S.u. Fn. 35.

5 BVerwGE 147, 312 (325, Rn. 46). Eingehend Masing, Die Mobilisierung des Bürgers für die Durchsetzung des Rechts, 1997, S. 19 ff., 175 ff., passim; ders., in: Hoffmann-Riem/Schmidt-Aßmann/Voßkuhle (Hrsg.), GVwR II, 2. Aufl. 2012, $\$ 7$ Rn. $88 \mathrm{ff}$.

6 Etwa durch die enge Auslegung des Art. 263 UAbs. 4 AEUV i.S.d. sog. PlaumannFormel: EuGH, Urt. v. 15.7.1963, C-25/62, Slg. 1963, 213 (238), ECLI:EU:C:1963:17 - Plaumann. Siehe jetzt speziell für eine europäische „Klimaklage": EuGH, Pressemitteilung Nr. 51/21 v. 25.3.2021, Rs. C-565/19. 
sche Wahrnehmung und Verteidigung gesamtgesellschaftlicher Interessen beruft.

Insbesondere im Bereich des - individualrechtlich eigentlich verödeten ${ }^{7}$ - Klimaschutzes versuchen Private und Verbände, vor Gericht ganz konkrete Maßnahmen im Kampf gegen den Klimawandel zu erstreiten ${ }^{8}$. Beim Bundesverfassungsgericht sind derzeit mehrere Verfassungsbeschwerden anhängig, die namentlich das neue Bundes-Klimaschutzgesetz für so unzureichend halten, dass dadurch der Staat seine objektiv-rechtlichen Schutzpflichten ${ }^{9}$ verletze $^{10}$. Die Deutsche Umwelthilfe, die bereits der Anordnung von Verkehrsverboten für Diesel-Kraftfahrzeuge in vielen Luftreinhalteplänen ( $\$ 47 \mathrm{BImSchG)}$ den Weg geebnet hat ${ }^{11}$, ringt unter ähnlichen Vorzeichen in gleich zwei Verfahren vor dem OVG Berlin-Brandenburg mit der Bundesregierung um mehr Klimaschutz u.a. im Straßenverkehr ${ }^{12}$.

Besonderes Aufsehen hat zuletzt außerdem die „Klimaklage“ dreier Landwirte mit ihren Familien und der Organisation Greenpeace gegen die Bundesregierung vor dem Verwaltungsgericht Berlin erregt ${ }^{13}$. Den Klägern war, gestützt insbesondere auf die objektiv-rechtliche Staatszielbestimmung des Art. 20a GG ${ }^{14}$, an der Feststellung gelegen, dass die Bundesregierung

7 Explizit z.B. $\$ 4$ Abs. 1 S. 7 Bundes-Klimaschutzgesetz. Siehe zur möglichen grundrechtlichen Fundierung die Nachweise in Fn. 33.

8 Guter Überblick über die vielgesichtigen „Klimaklagen“ bei Wegener, ZUR 2019, 3 (6 ff.); Bickenbach, JZ 2020, 168 ff.

9 Zur Einordnung der grundrechtlichen Schutzpflichten in den Bereich des objektiven Rechts statt aller und m.w.N. Dreier, in: ders. (Hrsg.), GG I, 3. Aufl. 2013, Vorb. Rn. 94, 102.

10 Siehe jetzt BVerfG, Beschl. v. 24.3.2021, 1 BvR 2656/18.

11 Schink/Fellenberg, NJW 2018, 2016 ff.; Winkler/Zeccola/Willing, DVBl. 2019, 79 ff.

12 Vgl. die Angaben und Klageschriften unter www.duh.de/klimaklagen/ $<20.2 .2021>$.

13 Das VG Berlin hat die Klage als unzulässig abgewiesen, wegen grundsätzlicher Bedeutung aber die Berufung zugelassen: VG Berlin, Urt. v. 31.10.2019, $10 \mathrm{~K}$ 412.18, Rn. 53 ff., juris.

14 Dass aus Art. 20a GG keine subjektiven Rechte folgen, ist an sich unbestritten: Salzwedel, in: Isensee/Kirchhof (Hrsg.), HStR IV, 3. Aufl. 2006, $\$ 97$ Rn. 25 ff., 32. Die Kläger haben einen „Schutzanspruch“ aber aus Art. 20a GG und den Grundrechten (u.a. Art. 2 Abs. 2 S. 1 und Art. 14 Abs. 1 GG) hergeleitet; vgl. zur vermeintlichen Rechtsverletzung die Klageschrift v. 25.10.2018, insbes. S. $61 \mathrm{ff}$, 74 ff., sowie die Replik vom 11.9.2019, S. 17 ff., abrufbar unter www.greenpeace.d e/sites/www.greenpeace.de/files/publications/20182710-greenpeace-guenther-klageschrift-klimaklage.pdf bzw. www.greenpeace.de/sites/www.greenpeace.de/files/publications/replik_final.pdf $<20.2 .2021>$. Siehe im Übrigen die Hinweise in Fn. 33 . 
zur Einhaltung ihrer durch Kabinettsbeschlüsse festgelegten Klimaziele und zur Ergreifung dazu geeigneter Maßnahmen verpflichtet sei.

Hinter diesen und ähnlichen Klagen stehen statt Altruismus ganz handfeste politische Interessen. Denn der Schutz von Umwelt und Klima ist Gegenstand intensiver und komplexer gesellschaftlicher Debatten, deren Auflösung sich, anders als es die Rechtsbehelfe suggerieren, als voraussetzungsvoll erweist. Zugleich lassen die maßgeblichen verfassungsrechtlichen Regelungen (hier: Art. 20a GG, ggf. „flankiert"15 durch besonders weitläufige Grundrechtstatbestände wie Art. 2 Abs. 2 S. 1 GG) der Konfliktbewältigung besonders viel Raum - angefangen schon bei der Frage, ob und inwieweit sich daraus überhaupt subjektive öffentliche Rechte und damit individuelle Klagerechte ergeben.

Wo die Einzelne auf den Ausgang dieser Debatten über den Rechtsweg Einfluss zu nehmen versucht, ist die (noch sehr unscharfe) Rede von Public Interest Litigation, Strategic Litigation oder spezieller von der Climate Change Litigation ${ }^{16}$. Die Gerichte sollen hier jeweils nur auf den ersten Blick Rechts-, im Kern aber eben politische Fragen entscheiden, die eingedenk der Vielzahl betroffener Interessen besonders aufgeladen sind. Diese Instrumentalisierung der Dritten Gewalt für politische Zwecke kann ebenso zweckmäßig wie gefährlich sein. Ihre Vorzüge und verfassungsrechtlichen wie praktischen Grenzen werden im Folgenden für die deutsche Verwaltungsgerichtsbarkeit skizziert.

15 Das vermeintliche Zusammenwirken der Normen ist dabei schon für sich genommen eine heikle Angelegenheit: Dreier (Fn. 9), Vorb. Rn. 156 ff.; Friedrich, Vom Recht zur Berechtigung, 2020, S. 234 ff. m.w.N.

16 Grundlegend schon Koch, Prozeßführung im öffentlichen Interesse, 1983. Aus der jüngeren Zeit z.B.: Ismer, Klimaschutz als Rechtsproblem, 2014, S. 37 ff.; Purnhagen, European Journal of Risk Regulation 2015, 443 f.; Saurer/Purnhagen, ZUR 2016, 16 ff.; Graser/Helmrich, Blick in die Wissenschaft 38 (2018), 43 ff.; weiterführend auch die Beiträge in dies. (Hrsg.), Strategic Litigation, 2019. Siehe aus dem internationalen Schrifttum etwa Peel/Osofsky, Climate Change Litigation, 2015, insbes. S. 4 ff., 35 ff.; Bouwer, Journal of Environmental Law 30 (2018), 483 ff. Vgl. zu einem aktuellen Fall aus den Niederlanden außerdem Voland, NVwZ 2019, 114 (114 f.); Wegener (Fn. 8), S. 3 ff. 


\section{Zweckmäßigkeit von „politischer Litigation“}

\section{Kompensation normativer Regelungs- und Vollzugsdefizite}

Die Inanspruchnahme gerichtlicher Hilfe in hochpolitischen Angelegenheiten reagiert zunächst ganz offenkundig auf strukturelle - oder jedenfalls empfundende - Regelungs- und Vollzugsdefizite sowie auf die Unfähigkeit oder Unwilligkeit anderer staatlicher Akteure, bestimmten Gefahren oder sogar Rechtsbrüchen mit der nötigen Entschlossenheit entgegenzutreten.

Soweit die jeweiligen Gefahren wie im Falle des Klimawandels - etwa für Leben und Gesundheit (Art. 2 Abs. 2 S. 1 GG) und für das Eigentum (Art. 14 Abs. 1 GG) - rechtlich bestenfalls unvollständig eingehegt sind und es schon an grundlegenden gesetzlichen Vorgaben fehlt, hinterlässt der (parlamentarische) Gesetzgeber selbst das „Vakuum“17, das die Gerichte füllen sollen ${ }^{18}$. Im Übrigen richtet sich der Vorwurf überwiegend an die Verwaltung, die vorhandene Regelungen zum Schutz der betroffenen Rechte und Rechtsgüter nicht ausschöpft, nur unzureichend beachtet oder bisweilen völlig verkennt.

Das gilt wiederum exemplarisch für den Schutz von Klima und Umwelt. Hier agieren im Rahmen der Leistungsverwaltung - z.B. bei der Genehmigung von Vorhaben - zunächst die zuständigen Behörden selbst oft nachlässig und mit einer ausgeprägten Nonchalance für die gesetzlichen Maßstäbe (und gerichtlichen Vorgaben) ${ }^{19}$. Aber auch viele Private haben zu den maßgeblichen Vorschriften ein gelinde gesagt gestörtes Verhältnis, wenn sie diese ignorieren oder ganz bewusst umgehen - man denke nur an den Diesel-Abgasskandal, der regelrechte Unrechtskulturen in der deutschen Automobilindustrie offenbart hat ${ }^{20}$. Die verantwortlichen staatlichen Stellen können oder wollen einen strikten Vollzug dann auch im

17 Vgl. erneut Winkler/Zeccola/Willing (Fn. 11), S. 79 ff.

18 Siehe zu den aktuellen Entwicklungen in der deutschen Klimaschutzgesetzgebung Schlacke, EnWZ 2020, 355 (359 ff.); Scharlau u.a., NVwZ 2020, $1 \mathrm{ff}$.

19 Zu Vollzugsdefiziten im Umweltrecht schon früh Lübbe-Wolff, NuR 1993, 217 ff.; aus jüngerer Zeit z.B. Gärditz, NVwZ 2014, 1 (8); ferner Siegel, ZUR 2017, 451 (453 f.). Vgl. zu bewussten staatlichen Rechtsbrüchen in anderem Kontext besonders deutlich OVG Münster, Urt. v. 17.7.2019, 4 D 36/19.NE, Rn. 123, juris; OVG Münster, Beschl. v. 24.9.2020, 4 B 1336/20.NE, Rn. 19 ff., juris.

20 In diese Richtung etwa LG Köln, Urt. v. 12.4.2018, 24 O 287/17, Rn. 30, juris; Heese, JZ 2019, 429 (429). 
Wege der Eingriffsverwaltung nicht durchweg garantieren und leisten so indirekt Schützenhilfe.

Dahinter steckt nicht zwangsläufig böser Wille: Viele Regelungen sind schlicht nicht vollzugstauglich, viele Behörden sachlich und personell nur unzureichend ausgestattet ${ }^{21}$. Der Staat wirkt bei der Wahrnehmung seiner Aufgaben in vielerlei Hinsicht überfordert. Zudem machen äußere Zwänge oder Fehlanreize die angezeigte Reaktion oft gar nicht (politisch) opportun $^{22}$ - das Land Niedersachsen etwa profitiert über seine Anteile an VW an den Gewinnen des rechtsbrüchigen Unternehmens.

\section{Effektivierung durch Rechtsschutz}

Wo die Dritte Gewalt unter dieser Prämisse ihre Kontrollbefugnisse weit in den Bereich des objektiven Rechts und des Politischen hinein verlagert, erweitert sie unzweifelhaft die praktische Wirksamkeit der betroffenen Normen oder füllt sie gar verbleibende Regelungslücken aus.

Hier dürfte sich auch bestätigen, dass Rechtsnormen umso effektiver sind, je eher und je mehr ihre Verletzung vor Gericht geltend gemacht wird oder jedenfalls geltend gemacht werden kann ${ }^{23}$. Die Effektivierung wirkt entweder schon präventiv, weil Legislative und Exekutive eingedenk der Aussicht auf eine gerichtliche Auseinandersetzung den Weg des (objektiven) Rechts nun gar nicht erst verlassen, oder jedenfalls repressiv, weil die Judikative vermehrt Rechtsverstöße beseitigt bzw. die zuständigen Stellen dazu in die Pflicht nimmt.

Namentlich für das Umwelt- und Naturschutzrecht ist bereits in den siebziger Jahren ein solcher Zusammenhang zwischen der Möglichkeit und dem Umfang gerichtlichen Rechtsschutzes einerseits sowie der Gesetzmäßigkeit der Verwaltung andererseits nachgewiesen worden ${ }^{24}$. Basale ent-

21 Siehe zum Ganzen in anderem Kontext Friedrich (Fn. 15), S. 28 ff., 217 ff.

22 Vgl. zum fehlenden Vollzugsinteresse und anderen Gründen für Vollzugsdefizite Krüper, Gemeinwohl im Prozess, 2009, S. 257 f.

23 So insbesondere Stern, Staatsrecht III/1, 1988, S. 875; gleichsinnig Huber (Fn. 1), Art. 19 Rn. 332, 342; Buchheim, Actio, Anspruch, subjektives Recht, 2017, S. 97 ff.

24 Siehe z.B. Rehbinder/Burgbacher/Knieper, Bürgerklage im Umweltrecht, 1972, S. 24 ff.; Koch, NVwZ 2007, 369 ff.; Schlacke, Überindividueller Rechtsschutz, 2008, S. 228; Gärditz (Fn. 19), S. 8; Wegener, ZUR 2018, 217 (217). Vgl. auch BTDrs. 14/6378, S. 61. 
scheidungstheoretische Erkenntnisse ${ }^{25}$ dürften das auch für alle anderen Bereichen nahelegen ${ }^{26}$.

\section{Waffengleichheit und Kräftebündelung}

Diese Form der Normeneffektivierung kann ferner rechtliche, politische und soziale Machtgefälle ausgleichen und kompensieren. Zum einen bewirkt die gerichtliche Kontrolle einen Gleichlauf von "Schutzinteressen“ und „Nutzungsinteressen“ in Bezug auf die jeweils betroffenen Rechtsgüter. Wer natürliche Ressourcen für wirtschaftliche Interessen „nutzt“, kann und wird diese Interessen in aller Regel vor Gericht durchsetzen, wenn er durch die staatliche Gewalt an der Nutzung gehindert wird. Umwelt und Klima kommen dabei nur passiv als Abwägungsbelange zum Tragen. Das ändert sich, wenn Klägerinnen deren Schutz aktiv einfordern.

Zum anderen kann sie Rechtsschutzoptionen gerade dort erweitern bzw. „verdoppeln“, wo potentielle Klägerinnen ansonsten auf die individuelle Durchsetzung solcher Gegenrechte verzichten. Die einzelne Umweltoder Klimaschützerin etwa wirkt im Streit mit einem großen Industrieunternehmen nicht selten wie „David gegen Goliath“ und wird in aller Regel kaum bereit sein, die erheblichen Prozess- und Kostenrisiken zu schultern ${ }^{27}$. Soll das Recht dieser Kräfteasymmetrie nicht zum Opfer fallen, bedarf es einer Fürsprecherin, die Einzelinteressen strategisch bündeln und verstärken kann. Diese Rolle übernehmen immer häufiger Vereinigungen und Verbände, und zwar auch jenseits der gesetzlichen Verbandsklagen. Sie sind typischerweise gut organisiert, verfügen regelmäßig über Erfahrung und erhebliche finanzielle Schlagkraft und können ihre Interessen deshalb weitaus effektiver und mit längerem Atem verfolgen als Einzelpersonen.

25 Weiterführend im Rechtsschutzkontext van Aaken, KritV 86 (2003), 44 (50 ff.); allgemeiner Englerth/Towfigh, in: Towfigh/Petersen, Ökonomische Methoden im Recht, 2. Aufl. 2017, $\mathbb{8}$ S. 237 ff.; Chatziathanasiou, Verfassungsstabilität, 2019, S. $75 \mathrm{ff}$.

26 Skeptisch hingegen Weyreuther, Verwaltungskontrolle durch Verbände?, 1975, S. 12 ff.; ähnlich Redeker, ZRP 1976, 163 (165); Ziekow, NVwZ 2005, 263 (264).

27 Vgl. zur "rationalen Apathie“ von Privatpersonen in anderem Kontext Towfigh/ Chatziathanasiou, in: Schulte-Nölke u.a. (Hrsg.), Neue Wege zur Durchsetzung des Verbraucherrechts, 2017, S. 93 (99 ff.). 


\section{Politischer Druck durch Rechtsschutz, oder: Rechtswegopposition}

Gerichtliche Kontrolle garantiert aber eben nicht nur die Gesetzmäßigkeit der Verwaltung, sondern erlaubt die Einflussnahme auf den politischen wie demokratischen Prozess insgesamt. Rechtsbehelfe spiegeln insoweit auch bedenkliche Vertrauensverluste im Hinblick auf die Integrität anderer staatlicher Institutionen: Wo wie beim Klimaschutz die Handlungsunfähigkeit oder sogar -unwilligkeit etwa der Bundesregierung in Rede steht und noch dazu parlamentarische und föderale Opposition durch komplexe Mehrheitsverhältnisse und das Wirken „großer“ Koalitionen erschwert werden, liegt die Gestaltung gesellschaftlicher Verhältnisse über den Umweg der Gerichte - gleichsam als Rechtswegopposition - sogar nahe.

Die Klägerin kann über den Rechtsweg gesellschaftliche Debatten anstoßen, deren Inhalt und Verlauf nachhaltig prägen, politischen Druck ausüben sowie den eigenen Zielen und Vorstellungen von der optimalen Problemlösung Aufmerksamkeit, Gewicht und Nachdruck verleihen. Im Idealfall wird das angerufene Gericht den rechtlichen Rahmen für diese Debatten in ihrem Sinne formen, womöglich sogar rechtliche „Grenzpfosten" einschlagen und damit bestimmte Inhalte bzw. Mindeststandards der politischen Diskussion - und dem Mehrheitsgesetzgeber! - von vornherein entziehen. Im Übrigen wird die Klägerin ganz unabhängig von der gerichtlichen Sachentscheidung jedenfalls auf einen Beachtungserfolg setzen: Die mediale Berichterstattung über "high profile“-Verfahren kann andere staatliche Stellen in Erklärungsnot bringen und unter Zugzwang setzen und das eigene Lager mobilisieren.

\section{Verfassungsrechtliche und praktische Grenzen}

So sinnvoll und wünschenswert die gerichtliche Durchsetzung von Interessen der Allgemeinheit und des objektiven Rechts im Einzelfall danach auch erscheinen mag, so sehr bringt sie das System des gerichtlichen Rechtsschutzes und damit den Verfassungsstaat insgesamt an seine Grenzen. 
1. Art. 19 Abs. 4 S. 1 GG: Systementscheidung für den individuellen Rechtsschutz

Gemeinwohlklagen brechen zunächst mit der Entscheidung des Grundgesetzes für den individuellen Rechtsschutz ${ }^{28}$. Das gilt zumindest dort, wo subjektive öffentliche Rechte entweder der Klägerin gar nicht zustehen, zu ihren Gunsten erst mühsam konstruiert werden müssen ${ }^{29}$ oder vollständig hinter das vorgetragene Allgemeininteresse zurücktreten. Denn Art. 19 Abs. 4 S. 1 GG verbürgt entgegen historischer und aktueller Alternativen ${ }^{30}$ nicht Interessen- oder Rechts-, sondern Rechteschutz. Grundsätzlich setzen danach sowohl die Zulässigkeit des jeweiligen Rechtsbehelfs als auch seine Begründetheit über den bloßen Rechtsverstoß hinaus eine Rechtsverletzung voraus (siehe nur $\$ \$ 42$ Abs. 2, 113 Abs. 1 S. 1 VwGO).

Über die Zuweisung einer entsprechenden Rechtsmacht zur Einleitung gerichtlicher Kontrollverfahren ebenso wie über die ausnahmsweise ${ }^{31} \mathrm{Zu}$ lassung überindividueller Rechtsbehelfe entscheidet allein der parlamentarische Gesetzgeber ${ }^{32}$. Ein umfassendes Recht auf Gesetzesvollziehung kennt die deutsche Rechtsordnung nicht. Es folgt - anders als gerade die Klimaklagen unter Berufung auf Art. 20a und Art. 2 Abs. 2 S. 1 GG suggerieren - insbesondere nicht aus den grundrechtlichen Schutzpflichten ${ }^{33}$, schon weil dem Staat bei deren Erfüllung grundsätzlich ein großzügig zu bemessender Einschätzungs- und Gestaltungsspielraum zukommt.

28 S.o. Fn. 1.

29 Siehe in anderem Kontext Friedrich (Fn. 15), S. 245 ff. Für ein mögliches „Recht auf Klimaschutz" unten Fn. 33.

30 Guter Überblick bei Schwerdtfeger, Der deutsche Verwaltungsrechtsschutz unter dem Einfluss der Aarhus-Konvention, 2010, S. 52 ff.; Epiney, NVwZ 2014, 465 ff. Siehe speziell zu Frankreich Masing (Fn. 5), S. 83 ff., 196 ff.; Ringena/Römling, JuWissBlog Nr. 139/2020 v. 11.12.2020, www.juwiss.de/139-2020/ <20.2.2021>.

31 Zum Primat des individuellen Rechtsschutzes: Krebs, in: v. Münch/Kunig (Hrsg.), GG I, 6. Aufl. 2012, Art. 19 Rn. 64; Huber (Fn. 1), Art. 19 Rn. 347.

32 BVerfG, NVwZ 2012, 694 (695); BVerfGE 129, 1 (20 ff.).

33 Siehe aus der Debatte um eine subjektiv-rechtliche Fundierung des Umwelt- und Klimaschutzes etwa Steinberg, NJW 1996, 1985 (1987 ff.); Salzwedel (Fn. 14), $\$ 97$ Rn. 25 ff.; Krüper (Fn. 22), insbes. S. 166 ff., 201 ff.; Ekardt, NVwZ 2013, 1105 (1106 ff.); Voland (Fn. 16), S. 117 ff.; Schlacke (Fn. 3), \$4 Rn. 9 ff.; Bickenbach (Fn. 8), S. 170 f. Eingehend zur bloß abstrakten grundrechtlichen Handlungsverpflichtung des Staates Ismer (Fn. 16), S. 45 ff.; zuvor schon in diesem Sinne BVerfGE 53, 30 (57 ff.); E 56, 54 (73 ff.); BVerfG, NJW 1983, 2931 (2932); BVerfG, NJW 1998, 3264 (3265). 
Das deutsche Modell verliert seine Existenzberechtigung schließlich nicht dadurch, dass das - insoweit anders sozialisierte ${ }^{34}$ - Unionsrecht der deutschen Rechtsordnung in gewissem Umfang die Öffnung für überindividuelle Formen des Rechtsschutzes abverlangt. Denn selbst der Anwendungsvorrang des Unionsrechts gebietet lediglich bereichsspezifisch Funktionserweiterungen, nicht hingegen die vollständige Aufgabe einer grundlegenden verfassungsrechtlichen Richtungsentscheidung durch einen umfassenden „Funktionswandel“ der Dritten Gewalt ${ }^{35}$.

\section{Filterfunktion zum Schutz der Rechte Dritter und allgemeiner Rechtsschutzeffektivität}

Art. 19 Abs. 4 S. 1 GG entfaltet seine Filterfunktion zunächst im Interesse Dritter. Erstens bleibt auf diese Weise gerichtlicher Rechtsschutz für alle effektiv. Zweitens wird die Freiheit Dritter nur dort gerichtlicher Kontrolle unterworfen, wo andere Private ihrerseits in subjektiven Rechten betroffen sind. Denn schon die Einleitung gerichtlicher Verfahren ist für Dritte mit erheblichen Belastungen verbunden: Die Rechtsverteidigung selbst gegen eine offensichtlich unbegründete Klage kann „Freiheitsentfaltungschancen" zumindest verteuern und zeitlich verzögern"

Wo Dritte von ihrer Freiheit dem objektiven Recht zuwider Gebrauch machen, mag ihr Schutz intuitiv befremden. Aber auch andernorts verpflichtet die Rechtsordnung zur Duldung rechtswidriger Zustände. Namentlich Verjährungs- und Rechtsbehelfsfristen sowie die Unterscheidung zwischen Rechtmäßigkeit und Wirksamkeit staatlichen Handelns ${ }^{37}$ machen deutlich: Wo andere Belange entgegenstehen, kommt es dem Rechts-

34 S.o. Fn. 5.

35 Weiterführend aus dieser Debatte Gärditz, in: Ständige Deputation des Deutschen Juristentages (Hrsg.), Verhandlungen des 71. Deutschen Juristentages, Bd. I, 2016, S. D1 ff.; ders., NJW-Beilage 2016, 41 ff.; Wegener, JZ 2016, 829 ff.; Rennert, DVB1. 2017, 69 ff. Von „Funktionserweiterungen“ spricht etwa Schmidt-Aßmann, Das allgemeine Verwaltungsrecht als Ordnungsidee, 2. Aufl. 2006, S. 224.

36 Den begünstigten Dritten kosten selbst unbegründete Rechtsbehelfe Zeit, Geld und Nerven, am Ende womöglich gar die Begünstigung als solche (z.B. über SS 48, $50 \mathrm{VwVfG}$ ). Siehe zur Drittbelastung durch Klagen und Klagerechte Schmidt-Aßmann (Fn. 1), Art. 19 IV Rn. 271; Gärditz (Fn. 19), S. 6 (dort das Zitat im Text), 8; vgl. ferner schon BVerfGE 116, 135 (154f.).

37 Dazu Ruffert, in: Ehlers/Pünder (Hrsg.), Allgemeines Verwaltungsrecht, 15. Aufl. 2016, $\$ 22$ Rn. 1. Eingehend zum Ganzen Bumke, Relative Rechtswidrigkeit, 2004, S. $12 \mathrm{ff} ., 201 \mathrm{ff}$, passim. 
staat auf flächendeckende Rechtskontrolle und Rechtmäßigkeitsfetischismus ${ }^{38}$ gar nicht an. Er verfolgt Rechtmäßigkeit nicht als Selbstzweck, sondern zum Schutz individueller Freiheit.

Darin dürfte auch der entscheidende Unterschied zur Unionsrechtsordnung liegen, die absolute Legalität tatsächlich zunächst um ihrer selbst willen postuliert. Sie muss darauf uneingeschränkt Wert legen und dafür auch die Einzelne einspannen, will sie die eigene Autorität und Autonomie gegenüber dem nationalen Recht möglichst effektiv absichern ${ }^{39}$.

\section{Fragen demokratischer Legitimation und Grundsatz der Gewaltenteilung}

Vor allem aber wirkt Art. 19 Abs. 4 S. 1 GG im Verhältnis der verfassten Gewalten untereinander als Kompetenzschleuse, die aus der Summe rechtswidrigen Staatshandelns nur einen kleinen Teil überhaupt als Prozessstoff zulässt und umgekehrt diejenigen Rechtsakte herausfiltert, über die Gerichte nicht sinnvoll entscheiden können oder es nicht sollen ${ }^{40}$. Insoweit bahnt die populare Durchsetzung von Allgemeininteressen tektonische Verschiebungen an.

\section{a) Ausweitung judikativer Kompetenzen auf Kosten der Ersten und Zweiten Gewalt}

Der deutschen Rechtsordnung ist eine strikte Gewaltentrennung ebenso fremd wie die noch in Weimar mit Nachdruck postulierte Trennung von Politik und Recht bzw. von politischer Dezision und richterlicher Subsumtion. Das Grundgesetz selbst verschränkt die Gewalten auf vielfältige Weise

38 Vgl. in anderem Kontext Augsberg, in: Ehlers u.a. (Hrsg.), Aktuelle Entwicklungen des Rechtsschutzes und der Streitbeilegung im Außenwirtschaftsrecht, 2013, S. 189 (205).

39 Vielsagend EuGH, Urt. v. 5.2.1963, C-26/62, Slg. 1963, 3 (26), ECLI:EU:C:1963:1 - van Gend \& Loos: Angewiesenheit der Unionsrechtsordnung auf „[d]ie Wachsamkeit der an der Wahrung ihrer Rechte interessierten Einzelnen“. Siehe im Übrigen schon die Nachweise oben in Fn. 5.

40 Vgl. in anderem Kontext jetzt Möllers, Verfassungsblog v. 16.5.2020, www.verfas sungsblog.de/sollte-die-eu-kommission-deutschland-wegen-des-karlsruher-ultra-vires-urteils-verklagen-contra/ $<16.5 .2020>$. 
miteinander ${ }^{41}$ und betraut mit der Letztentscheidung über die Geltung von formellem Recht ein Gericht (Art. 93 und 100 GG), das noch dazu von „jedermann“ (Art. 93 Abs. 1 Nr. 4a GG) zu diesem Zweck angerufen werden kann. Dass Gerichte bisweilen Politik machen und Recht nicht lediglich sprechen ${ }^{42}$, kann in der Bundesrepublik also kaum mehr überraschen.

Umso wichtiger ist, dass die Gerichte sich dabei in Zurückhaltung üben und nicht durch die Ausweitung von Kompetenzen oder extensive Kontrolle dieses Kräftearrangement aus dem Gleichgewicht bringen. Gerade die in Art. 19 Abs. 4 S. 1 GG angelegte Differenzierung zwischen subjektiven Rechten und objektivem Recht erweist sich vor diesem Hintergrund als zentrale Kompetenzausübungsschranke ${ }^{43}$. Wo umgekehrt die Gerichte diese Sperre umgehen und statt subjektiver Rechte bloße Interessen schützen oder gar neue subjektive Rechte erfinden, erschließen sie sich auf Kosten der anderen Gewalten eine bedenkliche Gestaltungsmacht sowie Mitspracherechte in Angelegenheiten, die das Prozessrecht ihnen gerade verschließ $\mathrm{t}^{44}$.

\section{b) Funktions-, Leistungs- und Legitimationsgrenzen zum Schutz individueller Freibeit}

Gewaltenteilung dient freilich keinem Selbstzweck. Sie sichert demokratische Legitimation und ordnet demokratische Verantwortungs- und Rechtsfertigungslasten zum Schutz bürgerlicher Freiheit.

41 Näher v. Arnauld, ZParl. 32 (2001), 678 ff.; Möllers, Gewaltengliederung, 2005, S. $66 \mathrm{ff} ., 410 \mathrm{ff}$; aus der jüngeren Rechtsprechung z.B. BVerfGE 128, 193 (209f.); E 139, 321 (361 ff., Rn. 125 ff.).

42 Eingehend im ersten Zugriff Jestaedt, in: Bumke (Hrsg.), Richterrecht zwischen Gesetzesrecht und Rechtsgestaltung, 2012, S. 50 ff.; Payandeh, Judikative Rechtserzeugung, 2017, insbes. S. 220 ff. Zu Zulässigkeit und Grenzen BVerfGE 128, 193 (209 ff.); E 132, 99 (127 ff.); BVerfG, Beschl. v. 6.6.2018, 1 BvL 7/14 u.a., Rn. 72 ff., juris.

43 Treffend insoweit die Mahnung von Schenke, in: Kahl/Waldhoff/Walter (Hrsg.), Bonner Kommentar zum GG, Art. 19 IV (Dezember 2009) Rn. 640 vor einem allzu beherzten Rückgriff der Justiz auf das Gebot effektiven Rechtsschutzes, der „ihren Machtanspruch pseudokonstitutionell bemäntel[e]“ (i.O. hervorgehoben).

44 Näher Möllers (Fn. 41), S. 157 ff.; aus dem neueren Schrifttum Steiger, VerwArch. 107 (2016), 497 (499 ff., insbes. 524 ff.); Friedrich (Fn. 15), S. 303 ff. m.w.N. 
aa) Arbeitsperspektive: Zwischen Abwägung, Anwendung und Erledigung

Die Aufgabenverteilung bildet zunächst eine den Gewalten jeweils eigene funktionelle Arbeitsperspektive ab. Der parlamentarische Gesetzgeber hat das "große Ganze“ im Blick und über die wesentlichen politischen Fragen zu entscheiden ${ }^{45}$. Das gilt insbesondere für komplexe Probleme, deren Lösung den Ausgleich einer Vielzahl kollidierender Rechte und Interessen erfordert. Dieser Ausgleich ist ureigene und erste Kompetenz des Parlaments ${ }^{46}$. Die Abgeordneten sind qua Amt Vertreter der Allgemeinheit (Art. 38 Abs. 1 S. 2 GG) und können für die Ermittlung und Abwägung der notwendigen Informationen auf dafür eigens ausgebildete Pfade und Beratungsmechanismen sowie auf entsprechende Ressourcen zurückgreifen.

Die rechtsprechende Gewalt ist innerhalb des vom Parlament gesetzten Rahmens nur zur Befriedung konkreter Streitfälle berufen. Sie operiert auf der Anwendungsebene grundsätzlich einzelfallbezogen. Schon die Informationsgewinnung vollzieht sich trotz Amtsermittlungsgrundsatz ( $\mathbb{8} 86$ Abs. 1 S. 1 VwGO) in viel engeren Bahnen als im parlamentarischen Prozess. Erst recht gilt das für die Willensbildung und Entscheidungsfindung: Dass die Beratung und Entscheidung ( $\mathbb{S} 192 \mathrm{ff}$. GVG, $\mathbb{} 43 \mathrm{DRiG}$ ) selbst der klügsten Kammer am Verwaltungsgericht anderen Regeln folgt als die Beschlussfassung im Deutschen Bundestag, liegt auf der Hand. Zur abschließenden Ermittlung, Abwägung und Durchsetzung von Belangen der Allgemeinheit ist die Justiz jedenfalls faktisch gar nicht in der Lage:

Zum einen zwingt „die kleine[n] Münze gerichtlicher Verfahren“47 zum Fokus auf die punktuelle Rechtskontrolle, ohne dass dabei politische Konflikte in ihrer Ganzheit in den Blick genommen werden könnten. In der Regel handelt es sich ja gerade um kontradiktorische Verfahren, die lediglich die Perspektiven der Beteiligten ( $\$ 63 \mathrm{VwGO}$ ) abbilden können, zumal auch nur diese durch die Entscheidung gebunden werden $(\mathbb{\$} 121 \mathrm{VwGO})$. Instrumente zur Erweiterung dieser Entscheidungsgrundlage um den not-

45 Lediglich exemplarisch BVerfGE 108, 282 (310 ff.); E 139, 19 (45 ff., Rn. 52 ff.); E 153, 1 (46, Rn. 101); aus dem unermesslichen Schrifttum zum Ganzen Lerche, in: Merten/Papier (Hrsg.), HGR III, 2009, $\$ 62$ Rn. 54 ff.; Schulze-Fielitz, in: Dreier (Hrsg.), GG II, 3. Aufl. 2015, Art. 20 (Rechtsstaat) Rn. 113 ff., 119 ff.

46 Siehe zu diesem Grundgedanken der parlamentarischen Demokratie statt aller Dreier (Fn. 9), Vorb. Rn. 67 ff., 134.

47 Treffend in anderem Kontext Wißmann, Verfassungsblog v. 6.2.2021, https://verfa ssungsblog.de/verfassungsbruch-schlimmer-ein-fehler/ $<20.2 .2021>$. 
wendigen gesamtgesellschaftlichen „Input“ stehen - von den nur eingeschränkt hilfreichen Möglichkeiten der Beiladung ( $\mathbb{S} 64 \mathrm{f}$. VwGO) und der Beiziehung des Vertreters des öffentlichen Interesses ( $\$ \$ 33 \mathrm{ff}$. VwGO) abgesehen - kaum zur Verfügung.

Zum anderen fehlt es ihr an der dafür notwendigen personellen und sachlichen Ausstattung. Die zur Entscheidung berufenen Personen sind zur Berücksichtigung aller relevanten Interessen in der Regel weder ausgebildet noch sozialisiert - schon die Juristenausbildung schärft weniger den Blick über den eigenen Tellerrand als vielmehr die Fähigkeit zur schematischen Falllösung. Zeit und Mittel haben sie dafür erst recht nicht. Der von vielen Richterinnen internalisierte „Erledigungsdruck“48 ist gerade nicht auf die unter allen denkbaren Gesichtspunkten „beste“ Lösung abstrakter Probleme, sondern auf eine schnelle und pragmatische Bewältigung der eigenen Arbeitslast im konkreten Fall gerichtet. Funktionsgrenzen markieren hier also zugleich ganz praktische Leistungsgrenzen. Wo Gerichte diese abstreifen, überfordern und schaden sie sich im Zweifel selbst.

\section{bb) Entscheidungsfindung und demokratische Legitimation}

Mit der jeweiligen Perspektive hängt eng zusammen die Art und Weise der Entscheidungsfindung, die wiederum den Grad der demokratischen Legitimation und der gesellschaftlichen Akzeptanz der jeweiligen Entscheidung bestimmt ${ }^{49}$.

Konflikte um Fragen, die - wie beim Klimaschutz - das öffentliche Meinungsbild scheinbar kompromisslos spalten, kann nur der parlamentarische Gesetzgeber dauerhaft befrieden. Das Gesetzgebungsverfahren zielt zwar auf eine Entscheidung der Mehrheit (vgl. nur Art. 42 Abs. 2 S. 1, 77 Abs. 1 S. 1, 121 GG), ist dabei aber weniger auf Konfrontation denn auf Inklusion bedacht. Es schenkt den maßgeblichen Akteuren und ihren widerstreitenden Interessen Gehör, stellt Lösungsalternativen öffentlich (Art. 42 Abs. 1 S. 1 und Abs. 3, Art. 44 Abs. 1 S. 1 sowie Art. 52 Abs. 3 S. 3 GG) ${ }^{50}$ zur Diskussion und ist in aller Regel bemüht um einen nachhaltigen Kompro-

48 Dazu kritisch Wittreck, NJW 2012, 3287 (3289 ff.); weiterführend Meyer, in: Kämmerer/Kotzur (Hrsg.), v. Münch/Kunig GG II, 7. Aufl. 2021, Art. 98 Rn. 115 ff.

49 Zur Bedeutung von Akzeptanz Morlok/Michael, Staatsorganisationsrecht, 4. Aufl. 2019, Rn. 73 ff.; Chatziathanasiou (Fn. 25), S. 2 m.w.N.

50 Dazu grundlegend BVerfGE 131, 152 (204 ff., Rn. 112 ff.); E 137, 34 (73, Rn. 77). Aus dem Schrifttum lediglich exemplarisch Achterberg, Parlamentsrecht, 1984, \21 S. $561 \mathrm{ff}$. 
miss $^{51}$. Legitimation wird hier gerade auch durch die Möglichkeit von Opposition und durch Repräsentation gewährleistet (siehe erneut Art. 38 Abs. 1 S. 2 GG ${ }^{52}$. Selbst wer sich in einem Kompromiss nicht wiederfindet, kann sich zumindest mit der Aussicht trösten, dass Mehrheitsverhältnisse sich jederzeit ändern können.

Die rechtsprechende Gewalt mag ebenfalls eine Befriedungs- und Stabilisierungsfunktion erfüllen ${ }^{53}$. Sie bietet aber schlicht kein äquivalentes Forum, in dem wesentliche Grundfragen des Zusammenlebens unter Berücksichtigung des gesellschaftlichen Interessenpluralismus sinnvoll ausgefochten werden könnten. Gerichtliche Entscheidungen vollziehen sich nicht von unten nach oben, sondern umgekehrt von oben nach unten. Auf die-

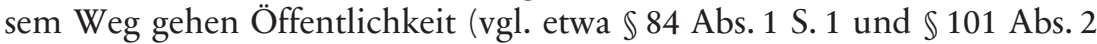
VwGO) und Sachverstand sowie nicht zuletzt politische Alternativen und Kompromisse verloren. Jedenfalls aber unterlaufen sie das parlamentarische Verfahren, das den öffentlichen Widerstreit kontroverser Positionen und deren Abbildung in der gefundenen Entscheidung garantiert. Selbst veränderte Mehrheitsverhältnisse lassen die Geltung einer gerichtlichen Entscheidung grundsätzlich unberührt.

Noch dazu schulden Richterinnen der Öffentlichkeit für ihre Tätigkeit in aller Regel keine echte Rechenschaft. Sie müssen jenseits der engen Grenzen des $₫ 339$ StGB und des $₫ 839$ Abs. 2 BGB selbst bei Fehlentscheidungen keine nennenswerten Konsequenzen und - anders als eine Parlamentsabgeordnete - auch nicht alle paar Jahre wieder um ihr Amt fürchten (vgl. nur Art. 97 Abs. 1 und 2 S. 1 GG).

All das birgt enormes Frustrationspotential: Wer vor Gericht verliert (oder nicht auf ganzer Linie gewinnt), nimmt diese Niederlage anders wahr als die Abstimmungsniederlage im Parlament - und wer an dem gerichtlichen Verfahren nicht beteiligt war, wird die Entscheidung ohnehin kaum akzeptieren, denn seine Interessen kamen darin gar nicht vor. Die Verlagerung politischer Debatten vom Parlament in die Gerichtssäle lässt also einen großen Teil der Gesellschaft von vornherein zurück. Die

51 BVerfGE 153, 1 (46, Rn. 101); BVerwG, Urt. v. 12.11.2020, 2 C 5.19, Rn. 22, juris. Zum Wert des Kompromisses in der Demokratie statt aller und m.w.N. Dreier, Kirche ohne König, 2020, S. 134.

52 Eingehend zur Repräsentation als Wesensmerkmal des parlamentarischen Systems Achterberg (Fn. 50), $\$ 5$ S. 76 ff.; Morlok, in: Dreier (Hrsg.), GG II, 3. Aufl. 2015, Art. 38 Rn. 32 ff.; zu den ideengeschichtlichen Hintergründen ferner Dreier, AöR 113 (1988), 450 (450 ff.).

$53 \mathrm{Zu}$ dieser Funktion der Rechtsprechung instruktiv Schmidt-Aßmann/Schenk, in: Schoch/Schneider/Bier (Hrsg.), VwGO, Einl. (Januar 2012) Rn. 165. 
Gerichte riskieren insoweit Enttäuschung auf allen Seiten und beschädigen im schlimmsten Fall sogar ihren eigenen Ruf ${ }^{54}$. Es liegt auf der Hand, dass sich der Anschein von politischem Aktionismus mit dem Anspruch auf unvoreingenommene Streitschlichtung nicht gut verträgt ${ }^{55}$.

cc) Aufgedrängtes Allgemeininteresse und judikativer Aktionismus als Kompromisshindernisse

Damit ist im Übrigen auch der Sache nicht gedient. Im Angesicht drängender Probleme mag das politische Engagement der Gerichte kurzfristig Abhilfe versprechen. Im schlimmsten Fall aber können ihre Entscheidungen Konflikte noch befeuern, notwendigen Debatten vorgreifen und binnengesellschaftliche Ausgleichsprozesse abwürgen - und so die Herausbildung tragfähiger Kompromisse zusätzlich erschweren.

Zuletzt standen etwa beim Klimaschutz der strategischen Hoffnung, mit gerichtlichen Klagen „die Welt zu retten“, emotionale Befürchtungen einer „Ökodiktatur über den Rechtsweg“ entgegen ${ }^{56}$. Wo ein Teil der Gesellschaft einem anderen Teil seine Lösung einfach überstülpt, rückt die Bewältigung gesamtgesellschaftlicher Herausforderungen in denkbar weite Ferne.

Für die Vereinigten Staaten hat derweil ausgerechnet Ruth Bader Ginsburg schon in den neunziger Jahren nachgezeichnet, dass die progressive Rechtsprechung des US Supreme Court in Bezug auf das Abtreibungsrecht von Frauen ebendiesem Anliegen im Rückblick eher abträglich war, weil sie zu früh zu weit gegangen ist und damit die eigentlich schon im Vordringen befindlichen Liberalisierungsbestrebungen auf Jahrzehnte hinaus ausgebremst hat ${ }^{57}$.

54 Entsprechende Sorgen äußert für den judikativen Klimaschutz mit Nachdruck Wegener (Fn. 8), S. 12 f.; gleichsinnig Bickenbach (Fn. 8), S. 177.

55 Vgl. aus der alten Debatte um den Jurisdiktionsstaat nur Rüthers, Die heimliche Revolution vom Rechtsstaat zum Richterstaat, 2014, insbes. S. 29 ff., 77 ff., passim.

56 Siehe zu diesem Spannungsverhältnis Steeger, taz.de v. 29.10.2019, www.taz.de/K1 agen-wegen-Klimawandel/!5634104/ <20.2.2021>.

57 Ginsburg, New York University Law Review 67 (1992), 1185 (1198ff.). 
dd) Grenzüberschreitung als faktisches Vollzugshindernis

Das Stichwort „Akzeptanz“ betrifft aber auch die praktische Umsetzung gerichtlicher Entscheidungen durch andere staatliche Stellen. Wo Gerichte sich politische Entscheidungsgewalt anmaßen, unterliegen sie auch politischen Rationalitäten, die sie bisweilen missverstehen. Hier büßen sie dann zwangsläufig diejenige Autorität ein, die normative Steuerung eigentlich voraussetzt. Es kann kaum verwundern, dass die Verwaltung immer häufiger gerichtliche Entscheidungen bewusst ignoriert ${ }^{58}$. Gerichte lindern dann nicht Vollzugsdefizite, sondern provozieren neue.

\section{Wer soll Hüter des Gemeinwohls sein?}

Die Artikulation noch so sympathischer öffentlicher Interessen im Rahmen des individuellen Rechtsschutzes leistet schließlich einer Privatisierung des Gemeinwohls Vorschub, die gesellschaftlichen Pluralismus unterschlägt. Der Begriff der „Public Interest Litigation“ erweist sich mindestens als irreführend, weil unter seinem Deckmantel letztlich doch nur Partikularinteressen geltend gemacht werden. Das aus der Diskussion um die gesetzliche Verbandsklage bekannte Problem ${ }^{59}$ wird hier noch zusätzlich verschärft, weil eine gesetzliche Grundlage gänzlich fehlt.

\section{a) „Das Gemeinwohl“?}

Schon in tatsächlicher Hinsicht bleibt die Redeweise von „dem Gemeinwohl“ oder „dem öffentlichen Interesse“ nebulös. In unserer komplexen, pluralistischen Gesellschaft können wir eigentlich nicht ohne Weiteres voraussetzen, dass bestimmte Anliegen - von gewissen „Grundverabredungen " 60 des freiheitlichen Verfassungsstaates einmal abgesehen (vgl. z.B. $\$ 4$ Abs. 2 BVerfSchG) - für jedermann auf Anhieb vollumfänglich zustimmungswürdig sind ${ }^{61}$.

58 Siehe aus der Diskussion nur Berkemann, DÖV 2019, 761 ff.; Will, VerwArch 110 (2019), $280 \mathrm{ff}$.

59 Stellvertretend und m.w.N. Krüper (Fn. 22), S. 157 ff.

60 Terminus bei Wißmann (Fn. 47).

$61 \mathrm{Im}$ ersten Zugriff Offe, in: Wingert/Günther (Hrsg.), Die Öffentlichkeit der Vernunft und die Vernunft der Öffentlichkeit, 2001, S. 459 (459). 
Im Übrigen zielt auch das Grundgesetz auf allgemeinen Konsens gar nicht ab: Demokratie meint nicht Herrschaft der Allgemeinheit, sondern Herrschaft der Mehrheit. Legitime Herrschaft der Mehrheit setzt allerdings den Schutz der Minderheit und ihrer Interessen voraus, und dass man diese als solche überhaupt wahr- und ernst nimmt. Das kann nicht gelingen, wenn die Mehrheit den Begriff des Gemeinwohls schlicht mit ihren eigenen Vorstellungen gleichsetzt und gegenläufige Anschauungen von vornherein ausblendet.

Im Gegenteil: Es ist Aufgabe demokratischer und speziell parlamentarischer Prozesse, verschiedene individuelle Interessen abzubilden und zum Ausgleich zu bringen ${ }^{62}$. Wo stattdessen der schillernde Topos des Gemeinwohls über Unterschiede hinweg- und falsche Einheit vortäuscht, wirkt er nicht inklusiv, sondern exklusiv. Seine Aktivierung riskiert die Verdrossenheit derjenigen, die sich nicht als Teil der jeweils ausgerufenen „Allgemeinheit" fühlen - und damit letztlich Spaltung der Gesellschaft.

\section{b) Einfallstore für Beliebigkeit statt belastbare Rechtsbegriffe}

Erst recht aber handelt es sich nicht um belastbare Rechtsbegriffe, die verbindliche Antworten der Gerichte auf politische Fragen tragen könnten. Im Gegenteil: Ihre Unbestimmtheit ${ }^{63}$ öffnet den Rechtsfindungsprozess für diffuse und beliebig austauschbare Moral- und Gerechtigkeitsvorstellungen der einzelnen Rechtsanwenderin. Jeder Versuch, darin bestimmte Vorgaben hineinzulesen, läuft Gefahr, den Gemeinwohltopos zu einem universalen Heilsversprechen für die Lösung gesellschaftlicher Konflikte zu überhöhen und ihm damit doch nur jede Strahlkraft zu nehmen.

Umso größere Vorsicht ist bei der Begründung gerichtlicher Entscheidungen und Kontrollbefugnisse geboten. Denn Inhalt und Ziel des gemeinen Wohls können nicht durch die Gerichte verordnet werden, sondern bestimmen sich im demokratischen Prozess von unten nach oben (Art. 20

62 Siehe statt aller Achterberg (Fn. 50), \$ 7 S. 108 ff.; Dreier, Hierarchische Verwaltung im demokratischen Staat, 1991, S. 26 ff.; sowie im Übrigen die Hinweise in Fn. 51 und Fn. 52.

63 Instruktiv zum Ganzen Leisner, DÖV 1970, 217 ff.; Isensee, in: ders./Kirchhof(Hrsg.), HStR III, 1. Aufl. 1988, 557 Rn. 4, 18; Häberle, Öffentliches Interesse als juristisches Problem, 2. Aufl. 2006, S. 32 ff.; Krüper (Fn. 22), S. 235 ff.; Wittreck, in: Gärditz (Hrsg.), VwGO, 2. Aufl. 2018, $\$ 36$ Rn. 6. Treffend außerdem schon Lubmann, Der Staat 1 (1962), 375 (375). 
Abs. 2 S. 1 GG $)^{64}$. Wer diesen Prozess prägen möchte, muss die notwendigen Mehrheiten erringen; vor Gericht hat er dagegen nichts verloren.

\section{c) Lobbyismus über den Rechtsweg? Public Interest Litigation als verkappte Special Interest Litigation}

Wo Private und private Verbände dennoch unter Berufung auf das Gemeinwohl Rechtsschutz suchen, fehlt jedenfalls ihnen selbst das zur Durchsetzung dieser Belange erforderliche demokratische Mandat ${ }^{65}$. Zwar ist privates Engagement im Interessenkreis der Allgemeinheit weder unüblich noch schlechthin schädlich ${ }^{66}$. Die Einflussnahme privater Akteure auf und ihre Einbindung in die Verwirklichung des Gemeinwohls sind sogar geboten, um gesellschaftlichen Pluralismus zu artikulieren und gegenläufige Interessen auszuloten. Gerade Verbände und Vereinigungen erweisen sich insoweit als wertvolle Bereicherung (siehe nur Art. 9 Abs. 1 und 3 sowie Art. 19 Abs. 3 GG; vgl. ferner 21 GG, Art. 140 GG i.V.m. Art. 137 WRV ${ }^{67}$. Insbesondere können sie bestehende Asymmetrien bei der Durchsetzung rechtlich geschützter Interessen kompensieren und denjenigen eine Stimme leihen, die im politischen Prozess ansonsten kein Gehör finden ${ }^{68}$.

Das gilt jeweils auch für den Rechtsschutz. Bedenken sind aber eben dort angezeigt, wo die einzelne Klägerin oder der klagende Verband von vornherein den Anspruch erhebt, verbindlich für die Allgemeinheit zu sprechen und deren Interessen wahrzunehmen. Denn grundsätzlich ist die Einzelne - anders als der Staat - nicht Sachwalterin der Allgemeinheit, sondern Partei für sich selbst.

64 Gleichsinnig Offe (Fn. 61), S. 485 ff.; Dreier, in: ders. (Hrsg.), GG II, 3. Aufl. 2015, Art. 20 (Republik) Rn. 22 m.w.N. Grundlegend zur Fließrichtung von Demokratie BVerfGE 20, 56 (99); E 44, 125 (140); E 138, 102 (109, Rn. 28).

65 Treffend speziell für die Klimaklagen wiederum Wegener (Fn. 8), S. 13.

66 Vgl. zu den diversen Erscheinungsformen von „Privatisierung“ Kämmerer, Privatisierung, 2001, S. 8 ff.; aus jüngerer Zeit Hansen, Indienstnahme und Verfassungstreue, 2020, S. $52 \mathrm{ff}$., passim.

67 Siehe nur BVerfGE 50, 290 (353 f.) zu Art. 9 GG.

68 Näher Grimm, in: ders., Die Zukunft der Verfassung, 1991, S. 241 (242 ff.); Schmidt-Aßmann (Fn. 35), S. 68 f.; Isensee, in: ders./Kirchhof(Hrsg.), HStR IX, 3. Aufl. 2011, $\mathbb{} 199$ Rn. 4 ff. 
Diese Annahme beruht weniger auf einer vermeintlich strikten Trennung von Staat und Gesellschaft ${ }^{69}$ oder einem staatlichen Gemeinwohlmonopol als vielmehr auf der individualistischen Konzeption grundrechtlicher Freiheit, die einer Operationalisierung der Einzelnen für die Allgemeinheit prinzipiell entgegensteht ${ }^{70}$ : Zum einen weil eine solche Vereinnahmung ihre Subjektsqualität letztlich aufhebt, zum anderen weil ihr selbst ein Recht nur auf den eigenen Lebensentwurf zusteht und nicht zugleich darauf, dass andere diesem folgen. Individuelle Freiheit macht die Einzelne weder zum Garanten noch zum Vertreter des Gemeinwohls. Hinzu kommt die Einsicht, dass nur der Staat überhaupt als „neutrale[r] Hüter“ und „,ehrlicher Makler“" für das Kollektiv in Betracht kommt und gerade darin seine Daseinsberechtigung findet (vgl. auch Art. 33 Abs. 4 $\mathrm{GG})^{71}$.

Speziell bei Verbänden, deren Bezeichnung z.B. als „Anwälte der Natur" über die Parteistellung Bände spricht ${ }^{72}$, sind Zweifel an der Unabhängigkeit und Uneigennützigkeit durchaus angebracht ${ }^{73}$. Denn Interessenbündelung führt unweigerlich zur Interessenintransparenz ${ }^{74}$. Tatsächlich betreiben Umweltverbände politischen Lobbyismus, der sich von der Aktivität anderer „pressure groups“ allenfalls unter Gesichtspunkten der öffentlichen Sympathie unterscheidet ${ }^{75}$. Wer ihre Rechtsbehelfe nur als Instru-

69 Weiterführend zur Debatte Michael, Rechtsetzende Gewalt im kooperierenden Verfassungsstaat, 2002, S. 247 ff.; Möllers, Staat als Argument, 2. Aufl. 2011, S. $228 \mathrm{ff}$.

70 Scharfe Kritik bei Gärditz (Fn. 35), S. 42; vgl. zuvor schon Krebs, in: Erichsen/ Hoppe/v. Mutius (Hrsg.), System des verwaltungsgerichtlichen Rechtsschutzes, 1985, S. 191 (194).

71 Zum zentralen Aspekt der Neutralität m.w.N. Calliess, NJW 2003, 97 (100 f.), der selbst aber staatliche Neutralitätslücken ausmacht; $v$. Danwitz, NVwZ 2004, 272 (274). Vgl. auch BVerfGE 33, 125 (159): Der Staat als „Hüter des Gemeinwohls gegenüber Gruppeninteressen“. Näher zum Ganzen Krüper (Fn. 22), S. 229 ff., $234 \mathrm{ff}$.

72 Klarstellung bei BVerwGE 104, 367 (370 f.). Vgl. aus der Diskussion um die Verbandsklage ferner Gassner, Treuhandklage zugunsten von Natur und Landschaft, 1984, S. 21 ff.; weiterführend jetzt Hansen (Fn. 66), S. 366 ff.

73 Skeptisch aus jüngerer Zeit z.B. Pagenkopf, NVwZ 2019, 185 (192 f.); eingehend ferner Spieth/Hellermann, NVwZ 2019, $745 \mathrm{ff}$.

74 Soldt, F.A.S. Nr. 29 v. 23.7.2017, 2; vgl. auch BGH, Urt. v. 4.7.2019, I ZR 149/18, Rn. 24 f., 30 ff., 40 ff., juris. Weiterführend zum Verhältnis von politischer Betätigung und Gemeinnützigkeit BFH, Urt. v. 10.1.2019, V R 60/17, Rn. 16 ff., juris sowie dazu Leisner-Egensperger, NJW 2019, $964 \mathrm{ff}$.

75 Wie hier kritisch wiederum Weyreuther (Fn. 26), S. 33 ff.; Pagenkopf (Fn. 73), S. 193. 
ment zur Kompensation von Verzerrungen im politischen Wettbewerb, als Ausdruck einer Re-Demokratisierung der Zivilgesellschaft und letztlich auch als Sicherung individueller Freiheit im Angesicht gesellschaftlicher und vor allem wirtschaftlicher Machtasymmetrien preist, übersieht zumindest, dass hier zugleich weitere Friktionen im Prozess der politischen Willens- und Mehrheitsbildung geschaffen werden.

Mit anderen Worten: Jede noch so gemeinnützige und mehrheitsfähige Klage dient letztlich der Durchsetzung gruppenegoistischer Partikularinteressen. In der Sache handelt es sich dann gerade nicht um Public Interest Litigation, sondern um Special Interest Litigation. Es gibt eigentlich keinen Anlass, diesen lebendigen Pluralismus durch das Bild vermeintlich unparteiischer Gemeinwohlklagen zu verschleiern. Schon der Versuch dürfte im Gegenteil der Akzeptanz etwaiger gerichtlicher Entscheidungen sowie auf lange Sicht dem gesellschaftlichen Zusammenhalt nur abträglich sein.

\section{d) Gemeinwohlklagen als Diskursvermeidungsstrategie?}

Wo sich Klägerinnen mit politischen Anliegen auf den Rechtsweg machen, bleibt schließlich der Verdacht, dass sie auf diese Weise echten Diskurs vermeiden oder sogar kurzschließen wollen. Es ist ja auch wahrlich bequemer, vor Gericht den eigenen Standpunkt zum Maß aller Dinge zu erheben, statt ihn ernsthaft zur Disposition zu stellen und sich mit gegenläufigen politischen Anschauungen überhaupt auseinanderzusetzen. Das gerichtliche Verfahren erregt dann zwar Aufmerksamkeit für eine wichtige Debatte, deren Ausgang die Klägerin selbst aber gar nicht abwarten will. Stattdessen soll das Gericht bereits Fakten schaffen und damit das Klagebegehren nicht nur rechtlich, sondern auch politisch mit einem besonderen Geltungsanspruch ,ausrüsten“.

Hier wird letztlich - wohl unter dem Eindruck vermeintlicher Vernunfthoheit - der Versuch unternommen, die eigenen Antworten auf hochkomplexe Fragen vorschnell mit dem Etikett der „Alternativlosigkeit“ zu versehen und damit jeden anderen Ansatz einem (zumindest moralischen) Zwang zur Rechtfertigung auszusetzen. Das verrät viel über die angebliche Konsensfähigkeit des eigenen Standpunkts - und spricht Bände 
über das Demokratieverständnis ausgerechnet eines Teils der Gesellschaft, der sich typischerweise für besonders liberal hält ${ }^{76}$.

\section{Zusammenfassung}

Die Idee, politischen Anliegen unter Berufung auf das Gemeinwohl über den Rechtsweg effektiv Nachdruck zu verleihen und auf diese Weise den gerichtlichen Rechtsschutz zu einem politischen Handlungsinstrument umzugestalten, mag im Einzelfall bestechen. Abseits echter subjektiver Rechtsbeeinträchtigungen hat sie jedoch im System des individuellen Rechtsschutzes keinen Platz. Sie bricht mit tragenden Grundprinzipien der gewaltenteiligen Demokratie und formuliert namentlich an die Dritte Gewalt eine unrealistische Erwartungshaltung, der diese faktisch nicht entsprechen kann und rechtlich nicht entsprechen darf. Noch dazu überhöht sie bloße Partikularinteressen zu einem vermeintlich vorrangigen „Public Interest", dessen nähere Bestimmung aber tatsächlich weder der einzelnen Klägerin noch dem angerufenen Gericht obliegt.

76 Vgl. zu diesem Selbstwiderspruch angeblich liberaler Eliten unlängst Kingreen, Verfassungsblog v. 7.4.2021, https://verfassungsblog.de/gehts-raus-und-spielts-fusb all $/<7.4 .2020>$. 